\title{
Periodic solutions of delay differential equations with feedback control for enterprise clusters based on ecology theory
}

\section{Ahmad Soltan Mohamadi, Abdolrasoul Pourabbas* and Seyed Mansour Vaezpour}

"Correspondence:

arpabbas@aut.ac.ir Department of Mathematics and

Computer Science, Amirkabir University of Technology, Hafez Ave., P.O. Box 15875-4413, Tehran, Iran

\begin{abstract}
By utilizing a fixed point theorem of strict-set-contraction, some criteria are established for the existence of positive periodic solutions of enterprise clusters based on ecology theory with time-varying delays and feedback controls.
\end{abstract}

Keywords: feedback control; strict-set-contraction; positive periodic solution; fixed point

\section{Introduction}

During recent years, many authors have worked on the existence of periodic solutions of delay differential equations with feedback control [1-6]. However, few papers have been published about the existence of these solutions of the aforementioned differential equations with feedback control for enterprise clusters based on ecology theory [7-13]. Zhou divided enterprise clusters into two models: the concentration of subcontractors around the dominant firm and the concentration of simple competitors, called the center halfback model and the net model [13]. Moreover, two models from biology were given and studied from an economic point of view. Accordingly, sufficient conditions were obtained to guarantee the co-existence and stability of enterprise clusters. Then, the developing strategy of enterprises was analyzed based on the logistic model, while the suggestions of constructing cooperative relation and choosing generalization or specialization tactics for the commodity were suggested [7]. In addition, based on a theoretical model of ecological population science, Wang and Pan made a detailed analysis of the equilibrium mechanism of enterprise clusters, including a net model and a center halfback model, and they drew the conclusion that the relationship of fierce competition and beneficial cooperation among enterprise clusters was the crucial factor to keep stability [11]. In a recent study the competition and cooperation system of two enterprises based on an ecosystem have been considered [9]:

$$
\left\{\begin{array}{l}
\frac{d x_{1}(t)}{d t}=r_{1}(t) x_{1}(t)\left(1-\frac{x_{1}(t)+\alpha\left(x_{2}(t)-c_{2}\right)^{2}}{K}\right), \\
\frac{d x_{2}(t)}{d t}=r_{2}(t) x_{2}(t)\left(1-\frac{x_{2}(t)-\beta\left(x_{1}(t)-c_{1}\right)^{2}}{K}\right),
\end{array}\right.
$$

where $x_{1}(t), x_{2}(t)$ are the output of enterprises $A$ and $B, r_{1}, r_{2}$ represent the intrinsic growth rate, $K$ is the carrying capacity of the market under natural unlimited conditions, $\alpha, \beta$

\section{Springer}

C2014 Soltan Mohamadi et al.: licensee Springer. This is an Open Access article distributed under the terms of the Creative Commons Attribution License (http://creativecommons.org/licenses/by/2.0), which permits unrestricted use, distribution, and reproduction in any medium, provided the original work is properly cited. 
denote the competitive coefficients of the two enterprises, $c_{1}, c_{2}$ are the initial production of the two enterprises. Therefore, the equation with non-constant coefficients is assumed, which can be got as a modified previous system with variable coefficients (letting $a_{1}=\frac{r_{1}}{K}$, $a_{2}=\frac{r_{2}}{K}, b_{1}=\frac{r_{1} \alpha}{K}, b_{2}=\frac{r_{2} \beta}{K}$ in previous system):

$$
\left\{\begin{array}{l}
\frac{d x_{1}(t)}{d t}=x_{1}(t)\left(r_{1}(t)-a_{1}(t) x_{1}(t)-b_{1}(t)\left(x_{2}(t)-c_{2}(t)\right)^{2}\right), \\
\frac{d x_{2}(t)}{d t}=x_{2}(t)\left(r_{2}(t)-a_{2}(t) x_{2}(t)-b_{2}(t)\left(x_{1}(t)-c_{1}(t)\right)^{2}\right) .
\end{array}\right.
$$

In real situations there is an undeniable time delay, and a long time delay may damage the stability of a system. Also the situation of enterprises is often distributed by unforeseen forces, which can lead to changes in the enterprises' parameters, such as intrinsic growth rates, therefore it is vitally important to study models with control variables which are the so-called disturbance functions [3,14-16]. Resuming the latest studies, the following competitive and cooperation model of $n$ satellite enterprises and a dominant enterprise under the center halfback model with time-varying delays and feedback controls is considered to find whether there is any periodic solution for this system:

$$
\left\{\begin{aligned}
\frac{d x_{i}(t)}{d t}= & x_{i}(t)\left[r_{i}(t)-a_{i}(t) x_{i}(t)-\sum_{j=1, j \neq i}^{n} b_{i j}(t) \frac{d x_{j}}{d t}\left(t-\tau_{j}(t)\right)\right. \\
& \left.-c(t)[y(t-\gamma(t))-d(t)]^{2}-e_{i}(t) u_{i}\left(t-\lambda_{i}(t)\right)\right] \\
\frac{d u_{i}(t)}{d t}= & -\alpha_{i}(t) u_{i}(t)-\sum_{j=1}^{n} \beta_{j}(t) x_{j}\left(t-\eta_{j}(t)\right) \\
\frac{d y(t)}{d t}= & y(t)\left[r(t)-a(t) y(t)-b(t) \frac{d y(t)}{d t}-\sum_{i=1}^{n} c_{i}(t)\left[x_{i}\left(t-\delta_{i}(t)\right)-d_{i}(t)\right]^{2}\right. \\
& -e(t) v(t-\lambda(t))] \\
\frac{d v(t)}{d t}= & -\mu(t) v(t)+v(t) y(t-\theta(t))
\end{aligned}\right.
$$

where $x_{i}(t), y(t)$ represent the output of the satellite enterprises, $A x_{i}$, and the core enterprise, $A y$, respectively, $r_{i}, r$ are the intrinsic growth rates, $a_{i}, a$ account for their respective self-regulations, $b_{i j}$ accounts for the rates of inter-enterprises $A x_{i}$ competition, $c$ represents the rate of intra-enterprise competition from $A y, c_{i}$ represents the rate of conversion of a commodity into the reproduction of enterprise $A x_{i}, d_{i}$ and $d$ represent the initial production of the enterprises, respectively, $u_{i}, v$ are the control variables, $i=1,2, \ldots, n$.

Let $R$ and $R^{+}$denote the set of all real numbers and nonnegative real numbers. Let $h$ be a continuous bounded function defined on $R$, and we set

$$
\bar{h}=\max \{h(t): t \in[0, w]\}
$$

and

$$
\underline{h}=\min \{h(t): t \in[0, w]\},
$$

where $h$ is a continuous $w$-periodic function.

In this paper we shall use the following hypotheses.

(H1) $r_{i}, r, a_{i}, a, b_{i j}, b, c_{i}, c, d_{i}, d, e_{i}, e, \alpha_{i}, \tau_{i}, \gamma, \lambda_{i}, \lambda, \delta_{i}, \eta_{i}, \mu, \theta$ are continuous $w$-periodic functions,

$$
\begin{aligned}
& p_{i}=e^{\int_{0}^{w} \alpha_{i}(s) d s}>1, \quad p=e^{\int_{0}^{w} \mu(s) d s}>1, \\
& q_{i}=e^{-\int_{0}^{w} r_{i}(s) d s}<1, \quad q=e^{-\int_{0}^{w} r(s) d s}<1,
\end{aligned}
$$




$$
\begin{aligned}
& G_{i}(t, s)=\frac{e^{\int_{t}^{s} \alpha_{i}(s) d s}}{e^{\int_{0}^{w} \alpha_{i}(s) d s}-1}, \quad G(t, s)=\frac{e^{\int_{t}^{s} \mu(s) d s}}{e^{\int_{0}^{w} \mu(s) d s}-1}, \\
& H(t, s)=\frac{e^{-\int_{t}^{s} r_{i}(s) d s}}{1-e^{-\int_{0}^{w} r_{i}(s) d s}}, \quad H(t, s)=\frac{e^{-\int_{t}^{s} r(s) d s}}{1-e^{-\int_{0}^{w} r(s) d s}}, \\
& B(t)=\max \left\{b_{i}(t): i=1, \ldots, n, b(t)\right\}, \\
& F_{i}(t)=\int_{t}^{t+w} G_{i}(t, s) \sum_{i=1}^{n} \beta_{i}(s) d s, \quad F(t)=v(t)=\int_{t}^{t+w} G(t, s) v(s) d s, \\
& M_{i 1}=\int_{0}^{w}\left(q_{i} a_{i}(s)+\sum_{i=1, i \neq j}^{n} b_{i j}(s)+q_{i} c(s)\|x\|_{1}+q_{i} e_{i}(s) F_{i}\left(s-\lambda_{i}(s)\right)\right) d s, \\
& M_{1}=\int_{0}^{w}\left[q a(s)+b(s)+q \sum_{i=1}^{n} c_{i}(s)\|x\|_{1}+q e(s) F(s-\lambda(s))\right] d s, \\
& M_{i 2}=\int_{0}^{w}\left(a_{i}(s)+\sum_{i=1, i \neq j}^{n} b_{i j}(s)+c(s)\|x\|_{1}+e_{i}(s) F_{i}\left(s-\lambda_{i}(s)\right)\right) d s, \\
& M_{2}=\int_{0}^{w}\left[a(s)+b(s)+\sum_{i=1}^{n} c_{i}(s)\|x\|_{1}+e(s) F(s-\lambda(s))\right] d s .
\end{aligned}
$$

$$
q_{i} a_{i}(t)\|x\|_{1}+q_{i} \sum_{i=1, i \neq j}^{n} b_{i j}(t)\|x\|_{1}+q_{i}^{2} c(t)\|x\|_{1}^{2}+q_{i} e_{i}(t) F_{i}\left(t-\lambda_{i}(t)\right)\|x\|_{1} \geq 0
$$

and

$$
q a(t)\|x\|_{1}+q b(t)\|x\|_{1}+q^{2} \sum_{i=1}^{n} c_{i}(t)\|x\|_{1}^{2}+q e(t) F\left(t-\lambda_{i}(t)\right)\|x\|_{1} \geq 0 .
$$

$$
\begin{aligned}
& \left(1+\underline{r}_{i}\right) \frac{q_{i}^{2}}{1-q_{i}} M_{i 1} \geq \max \left\{a_{i}(t)+\sum_{i=1, i \neq j}^{n} b_{i j}(t)+c(t)\|x\|_{1}+e_{i}(t) F_{i}\left(t-\lambda_{i}(t)\right): t \in[0, w]\right\}, \\
& (1+\underline{r}) \frac{q^{2}}{1-q} M_{1} \geq \max \left\{a(t)+b(t)+\sum_{i=1}^{n} c_{i}(t)\|x\|_{1}+e(t) F(t-\lambda(t)): t \in[0, w]\right\} .
\end{aligned}
$$

$(\mathrm{H} 4)$

$$
\begin{aligned}
& \left(1-\bar{r}_{i}\right) \frac{1}{q_{i}\left(1-q_{i}\right)} M_{i 2} \\
& \quad \leq \min \left\{q_{i} a_{i}(t)+q_{i} \sum_{i=1, i \neq j}^{n} b_{i j}(t)+q_{i} c(t)\|x\|_{1}+q_{i} e_{i}(t) F_{i}\left(t-\lambda_{i}(t)\right): t \in[0, w]\right\}, \\
& (1-\bar{r}) \frac{1}{q(1-q)} M_{2} \\
& \quad \leq \min \left\{q a(t)+q b(t)+q \sum_{i=1}^{n} c_{i}(t)\|x\|_{1}+q e(t) F(t-\lambda(t)): t \in[0, w]\right\} .
\end{aligned}
$$


In order to obtain our main results, we first make the following preparations.

Definition 1.1 Let $X$ be Banach space and $E$ be a closed, nonempty subset of $X ; E$ is said to be a cone if

(i) $\alpha u+\beta v \in E$, for all $u, v \in E$ and all $\alpha, \beta>0$,

(ii) $u,-u \in E$ imply $u=0$.

Let $X$ be a Banach space and $E$ be a cone in $X$. The semi-order induced by the cone $E$ is denoted by ' $\leq$.' That is, $x \leq y$ if and only if $y-x \in E$. For a bounded subset $A \subset X$, let $\alpha_{X}(A)$ denote the (Kuratowski) measure of non-compactness defined by

$$
\begin{aligned}
\alpha_{X}(A)= & \inf \left\{\delta>0 \text { : there is a finite number of subsets } A_{i} \subset A\right. \\
& \text { such that } \left.A=\bigcup_{i} A_{i} \text { and } \operatorname{diam}\left(A_{i}\right)=\delta\right\}
\end{aligned}
$$

where $\operatorname{diam}\left(A_{i}\right)$ denotes the diameter of the set $A_{i}$.

Definition 1.2 Let $E, F$ be two Banach spaces and $D \subset E$, a continuous and bounded map $T: D \rightarrow F$ is called $k$-set-contractive if for any bounded set $S \subset D$ we have

$$
\alpha_{F}(T(S)) \leq k \alpha_{E}(S)
$$

$T$ is called strict-set-contractive if it is $k$-set-contractive for some $0 \leq k<1$.

The following lemma is cited from $[17,18]$, which is useful for the proof of our main result of this paper.

Lemma 1.1 Let $P$ be a cone of the real Banach space $X$ and

$$
\Omega_{R}=\{x \in X:\|x\|<R\}, \quad \Omega_{r}=\{x \in X:\|x\|<r\},
$$

with $R>r>0$. Suppose that $T: P \cap \bar{\Omega}_{R} \backslash \Omega_{r} \rightarrow P$ is strict-set-contractive such that one of the following two conditions is satisfied:

(i) Not $T x \geq x \forall x \in P \cap \partial \Omega_{r}$.

(ii) Not $T x \leq x \forall x \in P \cap \partial \Omega_{R}$.

Then $T$ has at least one fixed point in $P \cap \bar{\Omega}_{R} \backslash \Omega_{r}$.

\section{Main results}

In this section, at first based on a fixed point theorem for the strict-set-contraction, we shall study the existence of at least one positive periodic solution of the system, then we state and prove our main results.

Now, each $w$-periodic solution of the equation

$$
\frac{d u_{i}(t)}{d t}=-\alpha_{i}(t) u_{i}(t)-\sum_{j=1}^{n} \beta_{j}(t) x_{j}\left(t-\eta_{j}(t)\right)
$$

and

$$
\frac{d v(t)}{d t}=-\mu(t) v(t)+v(t) y(t-\theta(t))
$$


is equivalent to that of the equations

$$
u_{i}(t)(t)=\int_{t}^{t+w} G_{i}(t, s) \sum_{j=1}^{n} \beta_{j}(s) x_{j}\left(s-\eta_{j}(s)\right) d s=\Phi_{x_{i}}(t)
$$

and

$$
v(t)=\int_{t}^{t+w} G(t, s) v(s) y(s-\theta(s)) d s=\Phi_{y}(t)
$$

Therefore, the existence problem of a $w$-periodic solution of system is equivalent to that of a $w$-periodic solution of the equations:

$$
\begin{aligned}
x_{i}(t)= & \int_{t}^{t+w} H_{i}(t, s) x_{i}(s)\left(a_{i}(s) x_{i}(s)+\sum_{j=1, i \neq j}^{n} b_{i j}(s) \frac{d x_{j}}{d s}\left(s-\tau_{j}(s)\right)\right. \\
& \left.+c(s)[y(s-\gamma(s))-d(s)]^{2}+e_{i}(s) \Phi_{x_{i}}\left(s-\lambda_{i}(s)\right)\right) d s,
\end{aligned}
$$

$i=1,2, \ldots, n$,

$$
\begin{aligned}
y(t)= & \int_{t}^{t+w} H(t, s) y(s)\left[a(s) y(s)+b(s) \frac{d y(s)}{d s}+\sum_{i=1}^{n} c_{i}(s)\left[x_{i}\left(s-\delta_{i}(s)\right)-d_{i}(s)\right]^{2}\right. \\
& \left.+e(s) \Phi_{y}(s-\lambda(s))\right] d s .
\end{aligned}
$$

In order to apply Lemma 1.1 to the equations, set

$$
C_{w}^{0}=\left\{x=\left[\begin{array}{c}
x_{1} \\
x_{2} \\
\vdots \\
x_{n} \\
y
\end{array}\right]: x_{i}, y \in C(R, R), x_{i}(t+w)=x_{i}(t), y(t+w)=y(t), t \in R\right\},
$$

with the norm defined by

$$
\|x\|=\max \left\{\max \left\{\left|x_{i}(t)\right|: t \in[0, w]\right\}, i=1,2, \ldots, n, \max \{|y(t)|: t \in[0, w]\}\right\}
$$

and

$$
C_{w}^{1}=\left\{x=\left[\begin{array}{c}
x_{1} \\
x_{2} \\
\vdots \\
x_{n} \\
y
\end{array}\right]: x_{i}, y \in C^{1}(R, R), x_{i}(t+w)=x_{i}(t), y(t+w)=y(t), t \in R\right\},
$$


with the norm defined by $\|x\|_{1}=\max \left\{\|x\|,\left\|x^{\prime}\right\|\right\}$. Then $C_{w}^{0}, C_{w}^{1}$ are of course Banach spaces. Define the cone $P$ in $C_{w}^{1}$ by

$$
P=\left\{x: x \in C_{w}^{1}, x_{i}(t) \geq q_{i}\|x\|_{1}, y(t) \geq q\|x\|_{1}, t \in[0, w]\right\} .
$$

Also for $R>r>0$, set

$$
\Omega_{R}=\left\{x \in C_{w}^{1}:\|x\|_{1}<R\right\}, \quad \Omega_{r}=\left\{x \in C_{w}^{1}:\|x\|_{1}<r\right\} .
$$

Let the map $T$ be defined by

$$
T x=\left[\begin{array}{c}
T x_{1} \\
T x_{2} \\
\vdots \\
T x_{n} \\
T y
\end{array}\right],
$$

where

$$
\begin{aligned}
T x_{i}(t)= & \int_{t}^{t+w} H_{i}(t, s) x_{i}(s)\left(a_{i}(s) x_{i}(s)+\sum_{j=1, i \neq j}^{n} b_{i j}(s) \frac{d x_{j}}{d s}\left(s-\tau_{j}(s)\right)\right. \\
& \left.+c(s)[y(s-\gamma(s))-d(s)]^{2}+e_{i}(s) \Phi_{x_{i}}\left(s-\lambda_{i}(s)\right)\right) d s,
\end{aligned}
$$

$i=1,2, \ldots, n$,

$$
\begin{aligned}
T y(t)= & \int_{t}^{t+w} H(t, s) y(s)\left[a(s) y(s)+b(s) \frac{d y(s)}{d s}+\sum_{i=1}^{n} c_{i}(s)\left[x_{i}\left(s-\delta_{i}(s)\right)-d_{i}(s)\right]^{2}\right. \\
& \left.+e(s) \Phi_{y}(s-\lambda(s))\right] d s .
\end{aligned}
$$

Lemma 2.1 Assume that (H1)-(H3) hold.

(i) If $\bar{r}_{i} \leq 1, \bar{r} \leq 1$, then $T: P \rightarrow P$ is well defined.

(ii) If (H4) holds and $\bar{r}_{i}>1, \bar{r}_{i}>1$, then $T: P \rightarrow P$ is well defined.

Proof (i) For any $x \in P$, it follows that $T x \in C_{w}^{1}$. Under the assumption (H2), for $t \in[0, w]$, we have

$$
\begin{gathered}
a_{i}(t) x_{i}(t)+\sum_{j=1, i \neq j}^{n} b_{i j}(t) \frac{d x_{j}}{d t}\left(t-\tau_{j}(t)\right)+c(t)[y(t-\gamma(t))-d(t)]^{2}+e_{i}(t) \Phi_{x_{i}}\left(t-\lambda_{i}(t)\right) \\
\geq q_{i} a_{i}(t)\|x\|_{1}+q_{i} \sum_{j=1, i \neq j}^{n} b_{i j}(t)\|x\|_{1}+q_{i}^{2} c(t)\|x\|_{1}^{2}+q_{i} e_{i}(t) F_{i}\left(t-\lambda_{i}(t)\right)\|x\|_{1} \geq 0
\end{gathered}
$$


and

$$
\begin{aligned}
& a(t) y(t)+b(t) \frac{d y(t)}{d t}+\sum_{i=1}^{n} c_{i}(t)\left[x_{i}\left(t-\delta_{i}(t)\right)-d_{i}(t)\right]^{2}+e(t) \Phi_{y}(t-\lambda(t)) \\
& \geq q a(t)\|x\|_{1}+q b(t)\|x\|_{1}+q^{2} \sum_{i=1}^{n} c_{i}(t)\|x\|_{1}^{2}+q e(t) F\left(t-\lambda_{i}(t)\right)\|x\|_{1} \geq 0
\end{aligned}
$$

moreover,

$$
\begin{aligned}
\max & \left\{\left|T x_{i}(t)\right|: t \in[0, w]\right\} \\
\leq & \max \left\{\frac { 1 } { 1 - q _ { i } } \int _ { t } ^ { t + w } x _ { i } ( s ) \left(a_{i}(s) x_{i}(s)+\sum_{i=1, i \neq j}^{n} b_{i j}(s) \frac{d x_{j}}{d s}\left(s-\tau_{j}(s)\right)\right.\right. \\
& \left.\left.+c(s)[y(s-\gamma(s))-d(s)]^{2}+e_{i}(s) \Phi_{x_{i}}\left(s-\lambda_{i}(s)\right)\right) d s: t \in[0, w]\right\} \\
= & \frac{1}{1-q_{i}} \int_{0}^{w} x_{i}(s)\left(a_{i}(s) x_{i}(s)+\sum_{i=1, i \neq j}^{n} b_{i j}(s) \frac{d x_{j}}{d s}\left(s-\tau_{j}(s)\right)\right. \\
& \left.+c(s)[y(s-\gamma(s))-d(s)]^{2}+e_{i}(s) \Phi_{x_{i}}\left(s-\lambda_{i}(s)\right)\right) d s
\end{aligned}
$$

and

$$
\begin{aligned}
\max & \{|T y(t)|: t \in[0, w]\} \\
\leq & \max \left\{\frac { 1 } { 1 - q } \int _ { t } ^ { t + w } y ( s ) \left(a(s) y(s)+b(s) \frac{d y(s)}{d s}\right.\right. \\
& \left.\left.+\sum_{i=1}^{n} c_{i}(s)\left[x_{i}\left(s-\delta_{i}(s)\right)-d_{i}(s)\right]^{2}+e(s) \Phi_{y}(s-\lambda(s))\right) d s: t \in[0, w]\right\} \\
= & \frac{1}{1-q} \int_{0}^{w} y(s)\left(a(s) y(s)+b(s) \frac{d y(s)}{d s}+\sum_{i=1}^{n} c_{i}(s)\left[x_{i}\left(s-\delta_{i}(s)\right)-d_{i}(s)\right]^{2}\right. \\
& \left.+e(s) \Phi_{y}(s-\lambda(s))\right) d s .
\end{aligned}
$$

Therefore, for $t \in[0, w]$, we obtain

$$
\begin{aligned}
T x_{i}(t) \geq & \frac{q_{i}}{1-q_{i}} \int_{t}^{t+w} x_{i}(s)\left(a_{i}(s) x_{i}(s)+\sum_{j=1, i \neq j}^{n} b_{i j}(s) \frac{d x_{j}}{d s}\left(s-\tau_{j}(s)\right)\right. \\
& \left.+c(s)[y(s-\gamma(s))-d(s)]^{2}+e_{i}(s) \Phi_{x_{i}}\left(s-\lambda_{i}(s)\right)\right) d s \\
= & \frac{q_{i}}{1-q_{i}} \int_{0}^{w} x_{i}(s)\left(a_{i}(s) x_{i}(s)+\sum_{j=1, i \neq j}^{n} b_{i j}(s) \frac{d x_{j}}{d t}\left(s-\tau_{j}(s)\right)\right.
\end{aligned}
$$




$$
\begin{aligned}
& \left.+c(s)[y(s-\gamma(s))-d(s)]^{2}+e_{i}(s) \Phi_{x_{i}}\left(s-\lambda_{i}(s)\right)\right) d s \\
\geq & q_{i}\|T x\|
\end{aligned}
$$

and

$$
\begin{aligned}
T y(t) \geq & \frac{q}{1-q} \int_{t}^{t+w} y(s)\left(a(s) y(s)+b(s) \frac{d y(s)}{d s}\right. \\
& \left.+\sum_{i=1}^{n} c_{i}(s)\left[x_{i}\left(s-\delta_{i}(s)\right)-d_{i}(s)\right]^{2}+e(s) \Phi_{y}(s-\lambda(s))\right) d s \\
= & \frac{q}{1-q} \int_{0}^{w} y(s)\left(a(s) y(s)+b(s) \frac{d y(s)}{d s}\right. \\
& \left.+\sum_{i=1}^{n} c_{i}(s)\left[x_{i}\left(s-\delta_{i}(s)\right)-d_{i}(s)\right]^{2}+e(s) \Phi_{y}(s-\lambda(s))\right) d s \\
\geq & q\|T x\| .
\end{aligned}
$$

Now we prove $T x_{i}(t) \geq q_{i}\left\|(T x)^{\prime}\right\|, T y(t) \geq q\left\|(T x)^{\prime}\right\|$, for $t \in[0, w]$; we find

$$
\begin{aligned}
\left(T x_{i}\right)^{\prime}(t)= & H_{i}(t, t+w) x_{i}(t+w)\left(a_{i}(t+w) x_{i}(t+w)\right. \\
& +\sum_{j=1, i \neq j}^{n} b_{i j}(t+w) \frac{d x_{j}}{d t}\left(t+w-\tau_{j}(t+w)\right) \\
& +c(t+w)[y(t+w-\gamma(t+w))-d(t+w)]^{2} \\
& \left.+e_{i}(t+w) \Phi_{x_{i}}\left(t+w-\lambda_{i}(t+w)\right)\right) \\
& -H_{i}(t, t) x_{i}(t)\left(a_{i}(t) x_{i}(t)+\sum_{j=1, i \neq j}^{n} b_{i j}(t) \frac{d x_{j}}{d t}\left(t-\tau_{j}(t)\right)\right. \\
& \left.+c(t)[y(t-\gamma(t))-d(t)]^{2}+e_{i}(t) \Phi_{x_{i}}\left(t-\lambda_{i}(t)\right)\right)
\end{aligned}
$$

and

$$
\begin{aligned}
(T y)^{\prime}(t)= & H(t, t+w) y(t+w)\left[a(t+w) y(t+w)+b(t+w) \frac{d y}{d t}(t+w)\right. \\
& +\sum_{i=1}^{n} c_{i}(t+w)\left[x_{i}\left(t+w-\delta_{i}(t+w)\right)-d_{i}(t+w)\right]^{2} \\
& \left.+e(t+w) \Phi_{y}(t+w-\lambda(t+w))\right] \\
& -H(t, t) y(t)\left[a(t) y(t)+b(t) \frac{d y}{d t}(t)+\sum_{i=1}^{n} c_{i}(t)\left[x_{i}\left(t-\delta_{i}\right)-d_{i}\right]^{2}\right.
\end{aligned}
$$




$$
\begin{aligned}
& \left.+e(t) \Phi_{y}(t-\lambda(t))\right]+r(t) T y(t) \\
= & r(t) T y(t)-y(t)\left[a(t) y(t)+b(t) \frac{d y}{d t}(t)+\sum_{i=1}^{n} c_{i}(t)\left[x_{i}\left(t-\delta_{i}\right)-d_{i}\right]^{2}\right. \\
& \left.+e(t) \Phi_{y}(t-\lambda(t))\right] .
\end{aligned}
$$

We have

$$
\left(T x_{i}\right)^{\prime}(t) \leq r_{i}(t) T x_{i}(t) \leq \bar{r}_{i} T x_{i}(t) \leq T x_{i}(t)
$$

and

$$
(T y)^{\prime}(t) \leq r(t) T y(t) \leq \bar{r} T y(t) \leq T y(t) .
$$

On the other hand, we find

$$
\begin{aligned}
-\left(T x_{i}\right)^{\prime}(t)= & x_{i}(t)\left(a_{i}(t) x_{i}(t)+\sum_{i=1, i \neq j}^{n} b_{i j}(t) \frac{d x_{j}}{d t}\left(t-\tau_{j}(t)\right)\right. \\
& \left.+c(t)[y(t-\gamma(t))-d(t)]^{2}+e_{i}(t) \Phi_{x_{i}}\left(t-\lambda_{i}(t)\right)\right)-r_{i}(t) T x_{i}(t) \\
\leq & \|x\|_{1}^{2}\left(a_{i}(t)+\sum_{i=1, i \neq j}^{n} b_{i j}(t)+c(t)\|x\|_{1}+e_{i}(t) F_{i}\left(t-\lambda_{i}(t)\right)\right)-r_{i}(t) T x_{i}(t) \\
\leq & \left(1+\underline{r}_{i}\right) \frac{q_{i}^{2}}{1-q_{i}}\|x\|_{1}^{2} \int_{0}^{w}\left(a_{i}(s)+\sum_{i=1, i \neq j}^{n} b_{i j}(s)+c(s)\|x\|_{1}+e_{i}(s) F_{i}\left(s-\lambda_{i}(s)\right)\right) d s,
\end{aligned}
$$

$-\underline{r}_{i} T x_{i}(t)$

$$
\begin{aligned}
& =\left(1+\underline{r}_{i}\right) \int_{t}^{t+w} \frac{q_{i}}{1-q_{i}} q_{i}\|x\|_{1}^{2}\left(a_{i}(s)+\sum_{i=1, i \neq j}^{n} b_{i j}(s)+c(s)\|x\|_{1}+e_{i}(s) F_{i}\left(s-\lambda_{i}(s)\right)\right) d s, \\
& -\underline{r}_{i} T x_{i}(t) \leq\left(1+\underline{r}_{i}\right) T x_{i}(t)-\underline{r}_{i} T x_{i}(t)=T x_{i}(t),
\end{aligned}
$$

and we have

$$
\begin{aligned}
-(T y)^{\prime}(t)= & y(t)\left[a(t) y(t)+b(t) \frac{d y}{d t}(t)+\sum_{i=1}^{n} c_{i}(t)\left[x_{i}\left(t-\delta_{i}\right)-d_{i}\right]^{2}+e(t) \Phi_{y}(t-\lambda(t))\right] \\
& -r(t) T y(t) \\
\leq & \|x\|_{1}^{2}\left[a(t)+b(t)+\sum_{i=1}^{n} c_{i}(t)\|x\|_{1}+e(t) F(t-\lambda(t))\right]-r(t) T y(t) \\
\leq & (1+\underline{r}) \frac{q^{2}}{1-q}\|x\|_{1}^{2} \int_{0}^{w}\left[a(s)+b(s)+\sum_{i=1}^{n} c_{i}(s)\|x\|_{1}+e(s) F(s-\lambda(s))\right] d s \\
& -r(t) \operatorname{Ty}(t)
\end{aligned}
$$




$$
\begin{aligned}
= & (1+\underline{r}) \int_{t}^{t+w} \frac{q}{1-q} q\|x\|_{1}^{2}\left[a(s)+b(s)+\sum_{i=1}^{n} c_{i}(s)\|x\|_{1}+e(s) F(s-\lambda(s))\right] d s \\
& -\underline{r} T y(t) \\
\leq & (1+\underline{r}) T y(t)-\underline{r} T y(t)=T y(t) .
\end{aligned}
$$

So we know $\left\|(T x)^{\prime}\right\| \leq\|T x\|$, so $\|T x\|_{1}=\|T x\|$. We have

$$
\left(T x_{i}\right)(t) \geq q_{i}\|T x\|_{1}, \quad(T y)(t) \geq q\|T x\|_{1}, \quad t \in[0, w] .
$$

Hence $T x \in P$.

(ii) In view of the proof of (i) we need to prove that

$$
\left(T x_{i}\right)^{\prime}(t) \leq T x_{i}(t) \quad \text { and } \quad(T y)^{\prime}(t) \leq T y(t)
$$

We obtain

$$
\begin{aligned}
\left(T x_{i}\right)^{\prime}(t) \leq & -q_{i}\|x\|_{1}\left(a_{i}(t) x_{i}(t)+\sum_{j=1, i \neq j}^{n} b_{i j}(t) \frac{d x_{j}}{d t}\left(t-\tau_{j}(t)\right)+c(t)[y(t-\gamma(t))-d(t)]^{2}\right. \\
& \left.+e_{i}(t) \Phi_{x_{i}}\left(t-\lambda_{i}(t)\right)\right)+r_{i}(t) T x_{i}(t) \\
\leq & r_{i}(t) T x_{i}(t)+q_{i}^{2}\|x\|_{1}^{2}\left(a_{i}(t)+\sum_{j=1, i \neq j}^{n} b_{i j}(t)+q c(t)\|x\|_{1}+e_{i}(t) F_{i}\left(t-\lambda_{i}(t)\right)\right)
\end{aligned}
$$

and

$$
\begin{aligned}
(T y)^{\prime}(t) \leq & -q\|x\|_{1}\left[a(t) y(t)+b(t) \frac{d y}{d t}(t)+\sum_{i=1}^{n} c_{i}(t)\left[x_{i}\left(t-\delta_{i}\right)-d_{i}\right]^{2}+e(t) \Phi_{y}(t-\lambda(t))\right] \\
& +r(t) \operatorname{Ty}(t) \\
\leq & r(t) \operatorname{Ty}(t)+q^{2}\|x\|_{1}^{2}\left[a(t)+b(t)+\sum_{i=1}^{n} c_{i}(t)\|x\|_{1}+e(t) F(t-\lambda(t))\right]
\end{aligned}
$$

Lemma 2.2 Assume that (H1)-(H3) hold and $R_{\bar{B}}<1$.

(i) If $\bar{r}_{i} \leq 1, \bar{r} \leq 1$, then $T: P \cap \bar{\Omega}_{R} \backslash \Omega_{r} \rightarrow P$ is strict-set-contractive.

(ii) If (H4) holds and $\bar{r}_{i}>1, \bar{r}>1$, then $T: P \cap \bar{\Omega}_{R} \backslash \Omega_{r} \rightarrow P$ is strict-set-contractive.

Proof We only need to prove (i), since the proof of (ii) is similar. It is easy to see that $T$ is continuous and bounded. Now we prove that $\alpha_{C_{w}^{1}}(T(S)) \leq R_{\bar{B}} \alpha_{C_{w}^{1}}(S)$ for any bounded set $S \subset P \cap \bar{\Omega}_{R}$. Let $\eta=\alpha_{C_{w}^{1}}(S)$, then, for any positive number $\varepsilon<R_{\bar{B}} \eta$, there is a finite family of subset $S_{i} \subset S$ satisfying $S=\bigcup_{i} S_{i}$ with $\operatorname{diam}\left(S_{i}\right) \leq \eta+\varepsilon$. Therefore

$$
\|x-y\|_{1} \leq \varepsilon \quad \text { for any } x, y \in S_{i}
$$

As $S$ and $S_{i}$ are precompact in $C_{w}^{0}$, it follows that there is a finite family of subsets $S_{i j} \subset S_{i}$ satisfying $S_{i}=\bigcup_{j} S_{i j}$ and

$$
\|x-z\| \leq \varepsilon \quad \text { for any } x, z \in S_{i j} .
$$


In addition, for any

$$
x=\left[\begin{array}{c}
x_{1} \\
x_{2} \\
\vdots \\
x_{n} \\
y
\end{array}\right] \in S
$$

and $t \in[0, w]$ we have

$$
\begin{aligned}
\left|\left(T x_{i}\right)(t)\right|= & \int_{t}^{t+w} H_{i}(t, s) x_{i}(s)\left(a_{i}(s) x_{i}(s)+\sum_{j=1, i \neq j}^{n} b_{i j}(s) \frac{d x_{j}}{d s}\left(s-\tau_{j}(s)\right)\right. \\
& \left.+c(s)[y(s-v(s))-d(s)]^{2}+e_{i}(s) \Phi_{x_{i}}\left(s-\lambda_{i}(s)\right)\right) d s \\
\leq & \frac{R^{2}}{1-q_{i}} \int_{0}^{w}\left(a_{i}(s)+\sum_{j=1, i \neq j}^{n} b_{i j}(s)+c(s) R+e_{i}(s) F_{i}\left(s-\lambda_{i}(s)\right)\right) d s:=M_{i 3}
\end{aligned}
$$

and

$$
\begin{aligned}
\left|\left(T x_{i}\right)^{\prime}(t)\right|= & \mid r_{i}(t) T x_{i}(t)-x_{i}(t)\left(a_{i}(t) x_{i}(t)+\sum_{j=1, i \neq j}^{n} b_{i j}(t) \frac{d x_{j}}{d t}\left(t-\tau_{j}(t)\right)\right. \\
& \left.+c(t)[y(t-v(t))-d(t)]^{2}+e_{i}(t) \Phi_{x_{i}}\left(t-\lambda_{i}(t)\right)\right) \mid \\
\leq & \bar{r}_{i} M_{i 3}+R^{2}\left(\bar{a}_{i}+\sum_{j=1, i \neq j}^{n} \bar{b}_{i j}+\bar{c}_{i} R+\frac{p_{i}}{p_{i}-1} \bar{e}_{i}\right) .
\end{aligned}
$$

Also we have

$$
\begin{aligned}
|(T y)(t)|= & \int_{t}^{t+w} H(t, s) y(s)\left[a(s) y(s)+b(s) \frac{d y}{d s}(s)+\sum_{i=1}^{n} c_{i}(s)\left[x_{i}\left(s-\delta_{i}(s)\right)-d_{i}(s)\right]^{2}\right. \\
& \left.+e(s) \Phi_{y}(s-\lambda(s))\right] d s \\
\leq & \frac{R^{2}}{1-q} \int_{0}^{w}\left[a(s)+b(s)+\sum_{i=1}^{n} c_{i}(s) R+e(s) F_{y}(s-\lambda(s))\right] d s:=M_{3}
\end{aligned}
$$

and

$$
\begin{aligned}
\left|(T y)^{\prime}(t)\right|= & \mid r(t) T y(t) \\
& +y(t)\left[a(t) y(t)+b(t) \frac{d y}{d t}(t)+\sum_{i=1}^{n} c_{i}(t)\left[x_{i}\left(t-\delta_{i}\right)-d_{i}\right]^{2}+e(t) \Phi_{y}(t-\lambda(t))\right] \mid \\
\leq & \bar{r} M_{3}+R^{2}\left(\bar{a}+\bar{b}+\sum_{i=1}^{n} \bar{c}_{i}+\frac{p}{p-1} \bar{e}\right) .
\end{aligned}
$$


Applying the Arzela-Ascoli theorem, we know that $T(S)$ is precompact in $C_{w}^{0}$. Then there is a finite family of subset $S_{i j k} \subset S_{i j}$ satisfying $S_{i j}=\bigcup_{k} S_{i j k}$ and

$$
\|T x-T z\| \leq \varepsilon \quad \text { for any } x, z \in S_{i j k}
$$

for any

$$
x=\left[\begin{array}{c}
x_{1} \\
x_{2} \\
\vdots \\
x_{n} \\
y
\end{array}\right], z=\left[\begin{array}{c}
z_{1} \\
z_{2} \\
\vdots \\
z_{n} \\
n
\end{array}\right] \in S_{i j k},
$$

we obtain

$$
\begin{aligned}
\max & \left\{\left|\left(T x_{i}\right)^{\prime}(t)-\left(T z_{i}\right)^{\prime}(t)\right|: t \in[0, w]\right\} \\
= & \max \left\{\mid r_{i}(t) T x_{i}(t)-r_{i}(t) T z_{i}(t)+x_{i}(t)\left(a_{i}(t) x_{i}(t)+\sum_{j=1, i \neq j}^{n} b_{i j}(t) \frac{d x_{j}}{d t}\left(t-\tau_{j}(t)\right)\right.\right. \\
& \left.+c(t)[y(t-v(t))-d(t)]^{2}+e_{i}(t) \Phi_{x_{i}}\left(t-\lambda_{i}(t)\right)\right)-z_{i}(t)\left(a_{i}(t) z_{i}(t)\right. \\
& +\sum_{j=1, i \neq j}^{n} b_{i j}(t) \frac{d z_{j}}{d t}\left(t-\tau_{j}(t)\right)+c(t)[n(t-v(t))-d(t)]^{2} \\
& \left.\left.+e_{i}(t) \Phi_{z_{i}}\left(t-\lambda_{i}(t)\right)\right) \mid: t \in[0, w]\right\} \\
\leq & \bar{r}_{i} \varepsilon+R\left(\bar{a}_{i} \varepsilon+\sum_{j=1, i \neq j}^{n} \bar{b}_{i j}(\varepsilon+\eta)+\bar{c}^{2}+\frac{p_{i}}{p_{i}-1} \bar{e}_{i} \varepsilon\right) \\
& +\varepsilon\left(\bar{a}_{i} R+\sum_{j=1, i \neq j}^{n} \bar{b}_{i j} R+\bar{c} R^{2}+\frac{p_{i}}{p_{i}-1} \bar{e}_{i} R\right)=R_{\bar{B}} \eta+M_{i 4} \varepsilon
\end{aligned}
$$

where $M_{i 4}=\bar{r}_{i}+2 R \bar{a}_{i}+2 R \sum_{j=1, i \neq j}^{n} \bar{b}_{i j}+2 R^{2} \bar{c}+2 R \frac{p_{i}}{p_{i}-1} \bar{e}_{i}$ and

$$
\begin{aligned}
\max & \left\{\left|(T y)^{\prime}(t)-(T n)^{\prime}(t)\right|: t \in[0, w]\right\} \\
= & \max \left\{\mid r(t) T y(t)-r(t) \operatorname{Tn}(t)+y(t)\left[a(t) y(t)+b(t) \frac{d y}{d t}(t)\right]\right. \\
& +\sum_{i=1}^{n} c_{i}(t)\left[x_{i}\left(t-\delta_{i}\right)-d_{i}\right]^{2}+e(t) \Phi_{y}(t-\lambda(t)) \\
& -n(t)\left[a(t) n(t)+b(t) \frac{d n}{d t}(t)+\sum_{i=1}^{n} c_{i}(t)\left[z_{i}\left(t-\delta_{i}\right)-d_{i}\right]^{2}\right. \\
& \left.\left.+e(t) \Phi_{n}(t-\lambda(t))\right] \mid: t \in[0, w]\right\}
\end{aligned}
$$




$$
\begin{aligned}
& \leq \bar{r} \varepsilon+R\left(\bar{a} \varepsilon+\bar{b}(\varepsilon+\eta)+\sum_{i=1}^{n} \bar{c}_{i} \varepsilon^{2}+\frac{p}{p-1} \bar{e} \varepsilon\right)+\varepsilon\left(\bar{a} R+\bar{b} R+\sum_{i=1}^{n} \bar{c}_{i} R^{2}+\frac{p}{p-1} \bar{e} R\right) \\
& =R_{\bar{B}} \eta+M_{4}^{\prime} \varepsilon,
\end{aligned}
$$

where $M_{4}^{\prime}=\bar{r}+2 R \bar{a}+2 R \bar{b}+2 R^{2} \sum_{i=1}^{n} \bar{c}_{i}+2 R \frac{p}{p-1} \bar{e}$ and $M_{4}=\max \left\{M_{4}^{\prime}, M_{i 4}\right\}$.

We have

$$
\|T x-T z\|_{1} \leq R_{\bar{B}} \eta+M_{4} \varepsilon \quad \text { for any } x, z \in S_{i j k} .
$$

As $\varepsilon$ is arbitrarily small, it follows that

$$
\alpha_{C_{w}^{1}}(T(S)) \leq R_{\bar{B}} \alpha_{C_{w}^{1}}(S)
$$

Therefore, $T$ is strict-set-contractive.

Theorem 2.1 Assume that (H1)-(H3) hold.

(i) If $\bar{r}_{i} \leq 1, \bar{r} \leq 1$, then the system has at least one positive w-periodic solution.

(ii) If (H4) holds and $\bar{r}_{i}>1, \bar{r}>1$, then system has at least one positive $w$-periodic solution.

Proof We only need to prove (i), since the proof of (ii) is similar. Let $R=\max \left\{\frac{1-q_{i}}{q_{i}^{2} M_{i 1}}, \frac{1-q}{q^{2} M_{1}}\right\}$ and $0<r<\min \left\{\frac{q_{i}\left(1-q_{i}\right)}{M_{i 2}}, \frac{q(1-q)}{M_{2}}\right\}$. Then we find $0<r<R$. It follows from Lemmas 2.1 and 2.2 that $T: P \cap \bar{\Omega}_{R} \backslash \Omega_{r} \rightarrow P$ is strict-set-contractive.

If there exists $\tilde{x} \in P \cap \partial \Omega_{r}$ or $\tilde{x} \in P \cap \partial \Omega_{R}$ such that $T \tilde{x}=\tilde{x}$ then the system has at least one positive $w$-periodic solution.

Now, we shall show that condition (ii) of Lemma 1.1 holds.

$T x \geq x$ so $T x-x \in P$, which implies that

$$
T x_{i}(t)-T x_{i}(t) \geq q_{i}\|T x-x\|_{1} \geq 0 \quad \text { for all } t \in[0, w] .
$$

We obtain

$$
\begin{aligned}
T x_{i}(t)= & \int_{t}^{t+w} H_{i}(t, s) x_{i}(s)\left(a_{i}(s) x_{i}(s)+\sum_{j=1, i \neq j}^{n} b_{i j}(s) \frac{d x_{j}}{d s}\left(s-\tau_{j}(s)\right)\right. \\
& \left.+c(s)[y(s-\gamma(s))-d(s)]^{2}+e_{i}(s) \Phi_{x_{i}}\left(s-\lambda_{i}(s)\right)\right) d s \\
= & \frac{1}{1-q_{i}}\|x\| \int_{0}^{w}\left(a_{i}(s)+\sum_{j=1, i \neq j}^{n} b_{i j}(s)+c(s) R+e_{i}(s) F_{i}\left(s-\lambda_{i}(s)\right)\right) d s<q_{i}\|x\| .
\end{aligned}
$$

We find

$$
\|x\| \leq\|T x\| \leq q_{i}\|x\| \leq\|x\|
$$

This is a contradiction. 
We only need prove that

Not $T x \leq x, \quad \forall x \in P \cap \partial \Omega_{R}$.

Suppose, for the sake of contradiction, that there exists $x \in P \cap \partial \Omega_{R}$ such that $T x>x$. Thus $T x-x \in P \backslash\{0\}$. Furthermore, for any $t \in[0, w]$, we have

$$
x_{i}(t)-T x_{i}(t) \geq q_{i}\|x-T x\|_{1} \geq 0 \quad \text { for all } t \in[0, w],
$$

and we obtain

$$
\begin{aligned}
T x_{i}(t)= & \int_{t}^{t+w} H_{i}(t, s) x_{i}(s)\left(a_{i}(s) x_{i}(s)+\sum_{j=1, i \neq j}^{n} b_{i j}(s) \frac{d x_{j}}{d s}\left(s-\tau_{j}(s)\right)\right. \\
& \left.+c(s)[y(s-\gamma(s))-d(s)]^{2}+e_{i}(s) \Phi_{x_{i}}\left(s-\lambda_{i}(s)\right)\right) d s \\
\geq & \frac{q_{i}}{1-q_{i}} q_{i}\|x\|_{1}^{2} \int_{0}^{w}\left(a_{i}(s)+\sum_{j=1, i \neq j}^{n} b_{i j}(s)+c(s) R+e_{i}(s) F_{i}\left(s-\lambda_{i}(s)\right)\right) d s .
\end{aligned}
$$

It follows that

$$
\|x\|>\|T x\|>R
$$

which is a contradiction. Therefore, conditions (i) and (ii) hold. By Lemma 2.1, we see that $T$ has at least one nonzero fixed point in $P \cap \bar{\Omega}_{R} \backslash \Omega_{r}$. Thus the system has at least one positive $w$-periodic solution. The proof of Theorem 2.1 is complete.

\section{Competing interests}

The authors declare that they have no competing interests.

\section{Authors' contributions}

The main idea of this paper was proposed by ASM. All authors contributed equally to the writing of this paper. All authors read and approved the final manuscript.

\section{Acknowledgements}

The authors are grateful to the referee for useful comments, which improved the manuscript, and for pointing out a number of misprints.

Received: 5 October 2013 Accepted: 25 June 2014 Published: 20 Aug 2014

\section{References}

1. Fan, G, Li, Y, Qin, M: The existence of positive periodic solutions for periodic feedback control systems with delays. Z. Angew. Math. Mech. 84(6), 425-430 (2004)

2. Gopalsamy, K, Weng, P: Global attractivity in a competition system with feedback controls. Comput. Math. Appl. 45, 665-676 (2003)

3. Huo, H, Li, W: Positive periodic solutions of a class of delay differential system with feedback control. Appl. Math. Comput. 148, 35-46 (2004)

4. Li, W, Wang, L: Existence and global attractivity of positive periodic solutions of functional differential equations with feedback control. J. Comput. Appl. Math. 180, 293-309 (2005)

5. Li, YK, Zhang, TW, Xing, ZW: The existence of nonzero almost periodic solution for Cohen-Grossberg neural networks with continuously distributed delays and impulses. Neurocomputing 73, 3105-3113 (2010)

6. Yin, F, Li, Y: Positive periodic solutions of a single species model with feedback regulation and distributed time delay. Appl. Math. Comput. 153(2), 475-484 (2004)

7. Guo, Q: Competitive strategies in an enterprise: an ecological model. Contemp. Econ. Manag. 27(2), 49-52 (2005) 
8. Li, WH, Han, FR: Studies on coordinated evolution laws between industry populations and imperial analysis. Chin. J. Manag. Sci. 12(5), 137-143 (2004)

9. Tian, XH, Nie, QK: On model construction of enterprises - interactive relationship from the perspective of business ecosystem. South. Econ. J. 4, 50-57 (2006)

10. Wang, BT, Shu, HY: Research on competition in mobile communications market: ecological model. J Beijing Union Univ., Nat. Sci. Ed. 20(2), 27-30 (2006)

11. Wang, Z, Pan, RY: Analysis to the equilibrium of industrial clusters based on ecological model. Stat. Thinktank 8, 32-35 (2008)

12. Zhang, R, Qian, XS, Gao, Z: A competitive model of enterprises based on ecology theory. Syst. Eng. 26, 116-119 (2008)

13. Zhou, H: Enterprises cluster co-existence model and stability analysis. Syst. Eng. 21(4), 32-37 (2003)

14. Liao, X, Ouyang, Z, Zhou, S: Permanence of species in nonautonomous discrete Lotka-Volterra competitive system with delays and feedback controls. J. Comput. Appl. Math. 211, 1-10 (2008)

15. Liu, P, Li, YK: Multiple positive periodic solutions of nonlinear functional differential system with feedback control. J. Math. Anal. Appl. 288, 819-832 (2003)

16. Zhang, TW, Li, YK, Ye, Y: Persistence and almost periodic solutions for a discrete fishing model with feedback control. Commun. Nonlinear Sci. Numer. Simul. 16, 1564-1573 (2011)

17. Cac, NP, Gatica, JA: Fixed point theorems for mappings in ordered Banach spaces. J. Math. Anal. Appl. 71, 547-557 (1979)

18. Guo, D: Positive solutions of nonlinear operator equations and its applications to nonlinear integral equations. Adv. Math. 13, 294-310 (1984)

10.1186/1029-242X-2014-306

Cite this article as: Soltan Mohamadi et al.: Periodic solutions of delay differential equations with feedback control

for enterprise clusters based on ecology theory. Journal of Inequalities and Applications 2014, 2014:306

\section{Submit your manuscript to a SpringerOpen ${ }^{\ominus}$ journal and benefit from:}

- Convenient online submission

- Rigorous peer review

Immediate publication on acceptance

- Open access: articles freely available online

- High visibility within the field

- Retaining the copyright to your article 